ఠ

ORIGINAL RESEARCH

\title{
MiR-699I-3p is identified as a novel suppressor in the expansion and activation of myeloid-derived suppressor cells in hepatoma-bearing mice
}

This article was published in the following Dove Medical Press journal:

OncoTargets and Therapy

\author{
Jin-Ping Sun* \\ Quan-Xing Ge* \\ Zheng Ren \\ Xin-Fang Sun \\ Shu-Ping Xie
}

Department of Gastroenterology, Huaihe Hospital of Henan University, Kaifeng 475000, China

*These authors contributed equally to this work
Correspondence: Quan-Xing Ge Department of Gastroenterology, Huaihe Hospital of Henan University, II 5 Ximen Street, Longting District, Kaifeng 475000, China

Tel/fax +86 37I 23906892

Email gequanxing_ga@I26.com
Objective: Myeloid-derived suppressor cells (MDSCs) are a heterogeneous group of cells derived from bone marrow, which has a significant ability in inhibition of immune cell response. In this study, the role of miR-6991-3p in regulating function of MDSCs was investigated.

Methods: MDSCs were isolated from different tissues of the control and hepatoma-bearing mice, and then expression of miR-6991-3p was detected with qPCR. Then, the miR-6991-3p mimic and inhibitor were respectively transfected into MDSCs, and behaviors of MDSCs were evaluated, including expansion, apoptosis, and production of inflammatory factors. Furthermore, we explored the underlying mechanism from which miR-6991-3p regulated MDSC functions. Results: Expression miR-6991-3p was markedly decreased in the MDSCs derived from spleen and further decreased in the MDSCs derived from the tumor tissue. MiR-6991-3p mimic transfection suppressed expansion and promoted apoptosis of MDSCs, accompanied by a significant decrease in the production of IL-6 and GM-CSF that are identified as stimulators in MDSC expansion. In contrast, miR-6991-3p inhibitor transfection displayed the opposite effect. miR6991-3p bound with and negatively regulated expression of LGALS9, a newly identified immune checkpoint gene and activator of STAT3, suppressing production of multiple factors that were customarily used to characterize the activation of MDSCs. MiR-6991-3p-accommodated MDSCs displayed less suppression on T cells, while miR-6991-3p inhibitor enhanced the suppression of MDSCs on T cells.

Conclusion: MiR-6991-3p is identified as a novel suppressor in the expansion and activation of myeloid-derived suppressor cells, which may be regarded as a promising target for modulating the function of MDSCs.

Keywords: hepatoma, myeloid-derived suppressor cells, miR-6991-3p, immunosuppression, galectin-9

\section{Introduction}

The occurrence and development of tumors are closely related to the dysfunction of the immune system in recognizing and clearing cancer cells, including blockade of proliferation and activation, increase of apoptosis, and impairment of functions in immune effector cells, as well as abnormality in number and function of T lymphocyte subsets. ${ }^{1}$ Cancer cells can inhibit the effective recognition and killing by the immune system, resulting in immune tolerance and thus promoting the occurrence and development of tumors. ${ }^{2}$ Tumor immunotherapy is a method of using immunological principles and methods to mobilize the natural defense mechanism or stimulate the body's own antitumor immune mechanism by artificial intervention, aiming to eliminate tumor cells in vivo. ${ }^{3}$ Immunotherapy for gastric cancer has attracted much attention because of its advantages 
such as low toxicity and good prognosis. ${ }^{4}$ To find an effective immunotherapy method is one of the main research directions of gastric cancer treatment at present and even in the future.

Myeloid-derived suppressor cells (MDSCs) are a group of heterogeneous cells derived from bone marrow, the phenotype of which, in mice, was $\mathrm{CD} 11 \mathrm{~b}^{+} \mathrm{Gr} 1^{+} .5$ They are immature precursors of dendritic cells, macrophages, and granulocytes. Under tumorigenesis and other pathological conditions, the maturation of MDSCs is blocked by cytokines, turning into immunosuppressive cells. ${ }^{6}$ In cancer patients, MDSCs are recruited, migrated, and amplified by proinflammatory cytokines, and the number and proportion of peripheral blood, immune organs, and cancer tissues are increased 10-fold or even tens of times. ${ }^{7}$ It is an important "umbrella" for tumor cells to escape recognition and killing of epidemic system. MDSCs mainly exert immunosuppressive function by producing Arg-1, iNOS, and ROS. ${ }^{8}$ Important premises for the immunosuppressive effect of MDSCs are their expansion and activation. Many factors could induce expansion and activation of MDSCs, including IL-6, GM-CSF, VEGF, IL-4/10/13, and TGF-beta, etc..$^{9,10}$

MiRNAs are functional small non-coding RNAs that regulate gene expression at the posttranscriptional level during various physiological and pathological processes. Recent evidence has revealed that miRNAs are involved in regulation of expansion and activation of MDSCs. For example, miR-30e was found distinctly upregulated in the MDSCs from a B-cell lymphoma mouse model, and it increased the differentiation capacity and immunosuppressive effect of MDSCs through targeting SOCS3, a negative regulator of the key regulatory pathway JAK2/STAT3 in expansion and activation of MDSCs. ${ }^{11}$ Another example is that hypoxiainducible expression of miR-10a and miR-21 promoted MDSC expansion and activation by targeting RORA and PTEN, and in vivo experiments showed that fewer MDSCs were generated in mice inoculated with miR-10a or miR-21 deficient glioma cells compared with those inoculated with normal glioma cells. ${ }^{12}$ Exploring the role of more microRNAs in expansion and function of MDSCs will be of great help in the development of immunotherapy for cancer.

Here, we found that, compared with the MDSCs derived from the blood of hepatoma-bearing mice, expression of miR-6991-3p was markedly decreased in the MDSCs derived from spleen and further decreased in the MDSCs derived from tumor tissue. Gain- and loss-of-function experiments were performed to investigate the role of miR-6991-3p in the expansion and immunosuppressive function of MDSCs in vitro. Then, we explored the potential mechanism from which miR-6991-3p affected MDSC behaviors.

\section{Materials and methods Mice}

Male C57BL/6 mice (approximately 6 weeks old) were purchased from Beijing Animal Center (Beijing, China) and maintained in a specific pathogen-free and controlled environment. Mice were injected with Hepa1-6 cells, which were obtained from Shanghai Institutes for Biological Sciences (Shanghai, China), using the high-pressure hydrodynamic method through the caudal vein $(2 \mathrm{~mL}$ of cell suspension containing $10^{6}$ cells were injected). Procedures were conducted 3 weeks later. All the procedures were conducted according to Institutional Animal Care and Use Committee of the Model Animal Research Center. The study was approved by the Ethics Committee of Huaihe Hospital of Henan University (China).

\section{Preparation of MDSCs and cell transfection}

MDSCs (CD11b ${ }^{+} \mathrm{Gr}^{+}$cells) were isolated using the same method reported elsewhere. Briefly, mice were euthanized by cervical dislocation, blood, spleen or tumor tissue was obtained, and then mononuclear cells were isolated by centrifugation and filtration. Then, MDSCs were preliminarily separated by density gradient centrifugation (orderly in PBS, 50\% Percoll, 60\% Percoll, 70\% Percoll, and 100\% Percoll). Subsequently, MDSCs were purified by staining with antibodies of CD11b and Gr1 followed by flow cytometry.

MDSCs were cultured in RPMI-1640 medium plus 10\% FBS. MiR-6991-3p mimics, inhibitors, and their negative controls (designed and synthesized by Ribobio Co., Ltd, Guangzhou, China) were transfected into the cells by using Lipofectamine 3000 Transfection Reagent (Thermo Fisher Scientific, Waltham, MA, USA) according to the manufacturer's instructions.

\section{Cell proliferation and apoptosis analyses}

Cell proliferation was evaluated by using the Cell Counting Kit-8 (CCK-8, Sigma-Aldrich Co., St Louis, MO, USA) assay which was carried out according to the manufacturer's instructions.

Cell apoptosis was detected by using the PI/Annexin V Cell Apoptosis Kit (Sigma-Aldrich Co.) according to the manufacturer's instructions. 


\section{Real-time qPCR}

Total RNA was isolated using Total RNA Isolation Kit (Tiangen, Beijing, China). Real-time qPCR reactions were carried out in a final volume of $25 \mu \mathrm{L}$ and run in $\mathrm{iQ}^{\mathrm{TM}} 5$ Multicolor Real-Time PCR Detection System (Bio-Rad Laboratories Inc., Hercules, CA, USA). The reactions were initially denatured at $95^{\circ} \mathrm{C}$ for 1 minute followed by 30 cycles of $95^{\circ} \mathrm{C}$ for 15 seconds and $55^{\circ} \mathrm{C}$ for 60 seconds. The data were calculated using $2^{-\Delta \Delta \mathrm{Ct}}$ method. Primers for miR6991-3p and U6 RNA (internal reference) were designed and produced by Ribobio Co., Ltd.

\section{3'UTR luciferase reporter assay}

The cDNA fragment corresponding to the LGALS9 mRNA and the mutated (mut) fragment were respectively produced by PCR and nest PCR. Then, they were respectively cloned into psiCHECK ${ }^{\mathrm{TM}}-2$ Vectors (Promega Corporation, Fitchburg, WI, USA). The constructs were confirmed by sequencing. Transfections were performed using Lipofectamine 3000 Transfection Reagent (Thermo Fisher Scientific) in the HEK293T cells. Either the wild type (wt) or the mut construct was co-transfected with $20 \mathrm{nM}$ of miR-6991-3p mimic or negative control (NC) mimic. Cells were harvested at 48 hours and the luciferase activity was measured using the DualGlo Luciferase Assay System (Promega Corporation).

\section{Western blotting}

An amount of $25 \mu \mathrm{g}$ of protein was separated by polyacrylamide gel and then electro-transferred to polyvinylidene fluoride membrane (EMD Millipore, Billerica, MA, USA) for immunoblotting analysis. The LGALS9 antibody (1:600, Abcam, Cambridge, UK), phosphorylated STAT3 (1:200, Abcam), and GAPDH murine antibody (1:800, Abcam) were respectively used to incubate with the membrane at $4^{\circ} \mathrm{C}$ overnight. After incubation with the appropriate HRPconjugate secondary antibody, the abundance of the proteins was detected using a ChemiDoc XRS Imaging System (Bio-Rad Laboratories Inc.).

\section{ROS and NO detection}

Oxidation-sensitive dye, DCFDA (Molecular Devices LLC, Sunnyvale, CA, USA/Thermo Fisher Scientific), was used to measure ROS production by MDSCs. Isolated MDSCs were simultaneously cultured with $2.5 \mu \mathrm{M}$ DCFDA and 30 $\mathrm{ng} / \mathrm{mL}$ phorbol myristate acetate (Sigma-Aldrich Co.) for 30 minutes. Analyses were then conducted by flow cytometry as described previously.
For NO production, the total $\mathrm{NO}$ in the cell lysate was measured using the Nitrate/Nitrite Assay Kit. Equal volumes of cell lysate $(60 \mu \mathrm{L}), 2 \mathrm{mM}$ NADPH $(5 \mu \mathrm{L}), \mathrm{FAD}(10 \mu \mathrm{L})$, and Nitrate Reductase $(5 \mu \mathrm{L})$ were incubated at $37^{\circ} \mathrm{C}$ for 30 minutes, followed by addition of $10 \mu \mathrm{L}$ of LDH Buffer and $\mathrm{LDH}$ solution. After incubation for 30 minutes at $37^{\circ} \mathrm{C}$, $50 \mu \mathrm{L}$ of Griess Reagent I and Griess Reagent II was added and it was incubated at room temperature for 10 minutes, absorbance was measured at $540 \mathrm{~nm}$. Nitrite concentrations were quantified by comparing the absorbance values to a standard curve generated by serial dilution of $100 \mu \mathrm{M}$ sodium nitrite.

\section{In vitro MDSC suppression assay}

To measure immunosuppressive function of "different treated" MDSCs, MDSCs transfected with various oligos were cocultured with $200 \mathrm{nM}$ OVA peptide (OVA257-264) in 96-well plates at a ratio of 1:0,1:1, and 1:4 for 48 hours. The production of IFN- $\gamma$ was measured by IFN- $\gamma$ ELISA Kit (4A Biotech, Beijing, China) according to the manufacturer's instructions.

\section{Statistical analyses}

Data were obtained from at least three independent experiments in every assay. Values were expressed as mean \pm SEM. Multiple comparisons were assessed by one-way ANOVA followed by Dunnett's tests with SPSS 23.0 software. Difference between groups was considered statistically significant if $P<0.05$.

\section{Results \\ MiR-699 I-3p expression was regulated by tumor microenvironment}

To investigate the number change of MDSCs in response to tumor microenvironment, MDSCs were respectively isolated from the spleen of normal mice and hepatoma-bearing mice. Our results showed that the proportion of MDSCs in the spleen of hepatoma-bearing mice was approximately ten times as high as that in the spleen of normal mice (Figure 1A). Then, MDSCs were respectively isolated from the blood, spleen, and tumor tissue, and the expression of miR-6991-3p was detected with qPCR. The results showed that compared with the blood, miR-6991-3p was significantly downregulated in MDSCs from the spleen and further downregulated in those from the tumor tissue (Figure 1B), suggesting that miR-6991-3p might be associated with tumor microenvironment. 

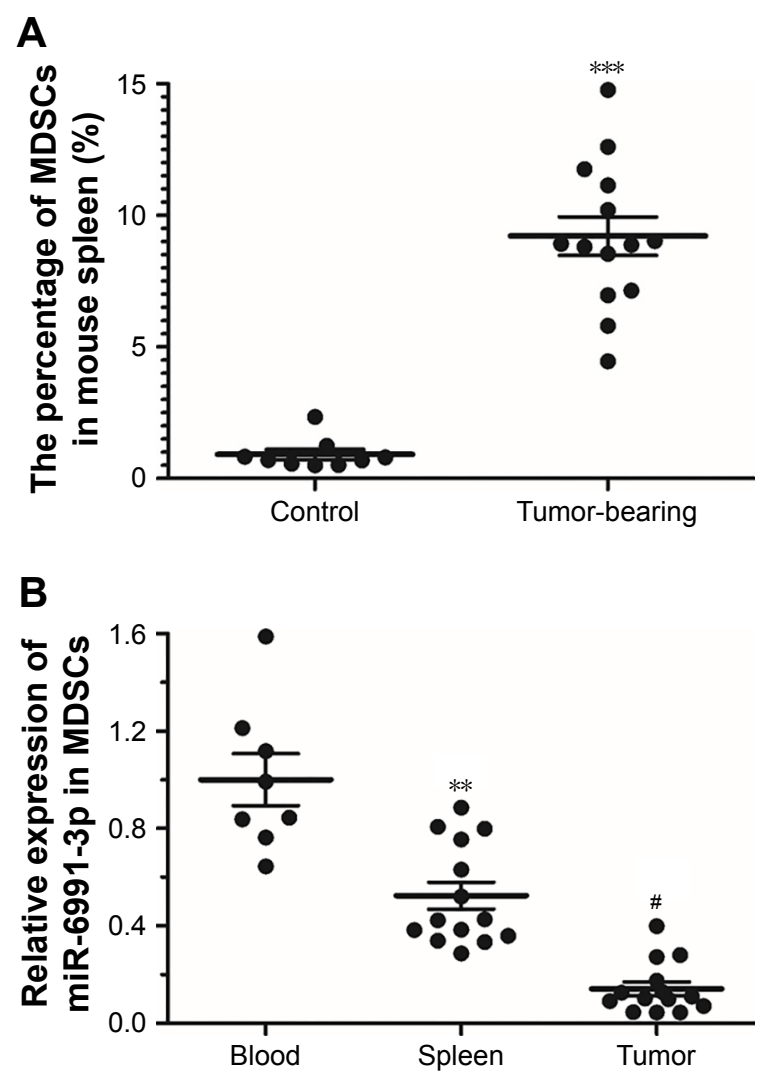

Figure I MiR-699|-3p expression was associated with tumor microenvironment. Notes: MDSCs were respectively isolated from the spleen of normal mice and hepatoma-bearing mice, and (A) the proportion of MDSCs occupied in the mononuclear cells was detected. MDSCs were isolated from the different parts of the hepatoma-bearing mice, and then (B), the expression of miR-699I-3p was detected with qPCR. $* * * P<0.001$ compared with control; $* * P<0.01$ compared with blood; ${ }^{\# P}<0.05$ compared with spleen.

Abbreviation: MDSCs, myeloid-derived suppressor cells.

\section{MiR-699I-3p suppressed expansion of MDSCs}

Then, to investigate the role of miR-6991-3p in the function of MDSCs, MDSCs were isolated and cultured in vitro, and then different concentrations of miR-6991-3p mimic or inhibitor were respectively transfected into the MDSCs. After incubation for 48 hours, the overexpression and interference efficiencies were confirmed by qPCR (Figure 2A and B). Amounts of $20 \mathrm{nM}$ miR-6991-3p mimic and $40 \mathrm{nM}$ miR-6991-3p inhibitor were applied in the following experiments. Our data from CCK-8 assay showed that miR-6991-3p mimic suppressed cell proliferation, while miR-6991-3p inhibitor promoted cell proliferation (Figure 2C). Moreover, miR-6991-3p mimic increased cell apoptosis, while miR-6991-3p inhibitor suppressed cell apoptosis (Figure 2D). Then, we detected the secretion of GM-CSF and IL-6 which could promote the expansion of MDSCs. The results showed that miR-6991-3p mimic suppressed the secretion of GM-CSF and IL-6, while miR6991-3p inhibitor promoted their secretion (Figure 2E and F).

\section{MiR-699I-3p directly targeted and negatively regulated expression of LGALS9, inhibiting the activation of STAT3}

Furthermore, the mechanism from which miR-6991-3p regulated the behavior of MDSCs was explored. We searched the potential target genes using bioinformatic tool. The results showed that the seed sequence of miR-6991-3p completely matched with seven consecutive bases at the 3'UTR of LGALS9 mRNA (Figure 3A). A wt LGALS9-3'UTR and a mut luciferase reporter vector was respectively constructed. The detailed information for the mut sequence was shown in Figure 3B. MiR-6991-3p significantly suppressed the luciferase activity of wt LGALS9-3'UTR luciferase reporter gene but could not change that of the mut LGALS9-3'UTR (Figure 3C). Western blotting analysis showed that overexpression of miR-6991-3p dramatically decreased and knockdown of miR-6991-3p increased the expression of LGALS9 protein (Figure 3D). Moreover, as an identified target of LGALS9, the activation of STAT3 was negatively regulated by miR-6991-3p (Figure 3D).

\section{MiR-699 I-3p suppressed the activation and immunosuppressive effect of MDSCs}

Finally, the effect of miR-6991-3p on the activation and immunosuppressive function of MDSCs was investigated. Our results showed that the secretion of IL-10 and IL-4, which could induce activation of MDSCs, was negatively regulated by miR-6991-3p (Figure 4A and B). Moreover, overexpression of miR-6991-3p significantly decreased and knockdown of miR-6991-3p increased the production of ROS and NO (Figure 4C and D). Finally, in order to determine the effect of miR-6991-3p on the immunosuppressive function of MDSCs, we cocultured OVA-specific OT-I T cells with naïve MDSCs, miR-6991-3p mimic transfected or miR-6991-3p inhibitor transfected MDSCs. The results showed that miR-6991-3p mimic transfection attenuated the suppressive effect of MDSCs on IFN- $\gamma$ secretion, while miR-6991-3p inhibitor transfection enhanced the suppressive effect of MDSCs on IFN- $\gamma$ secretion (Figure 4E).

\section{Discussion}

MiR-6991-3p was discovered by Ago2-based immunoprecipitation and RNA sequencing in $2012 .{ }^{13}$ Up to date, there 
A

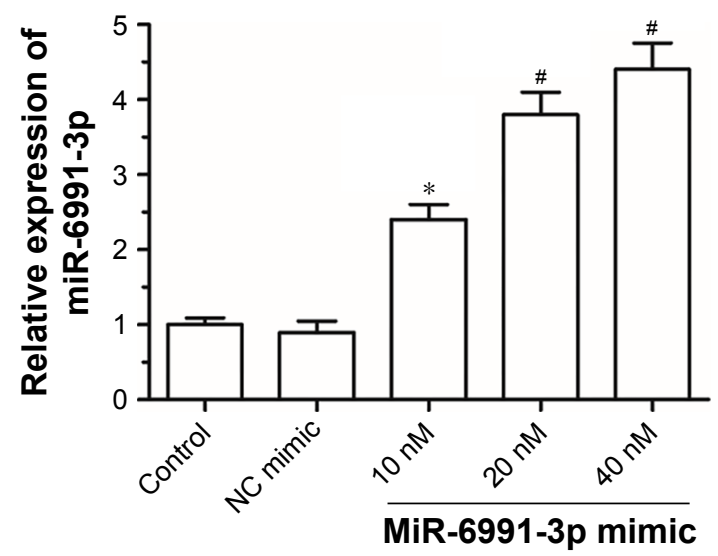

C

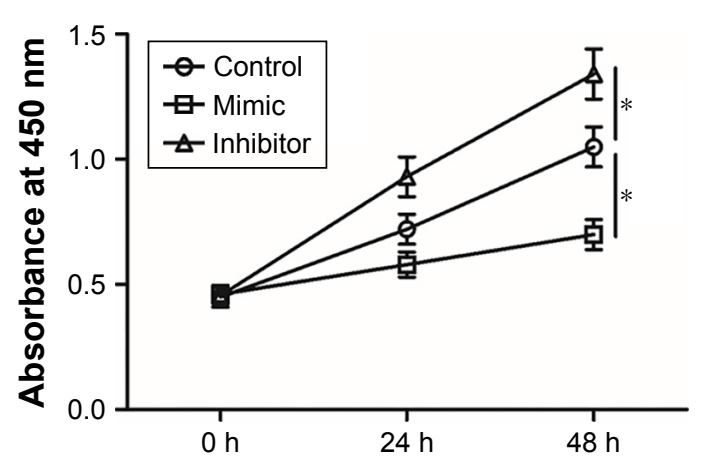

E

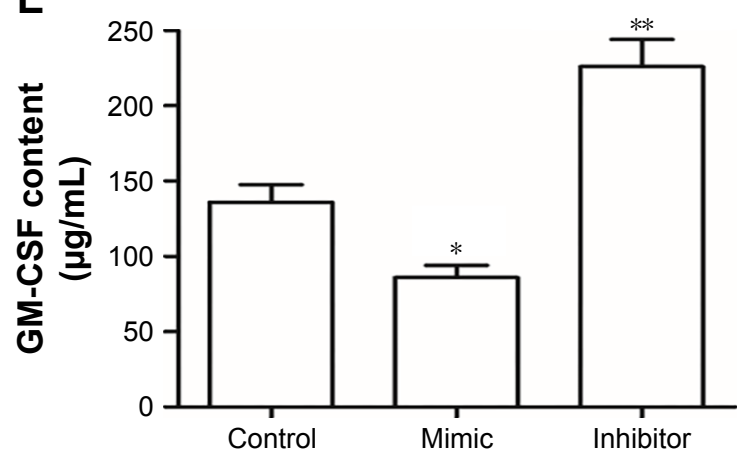

B
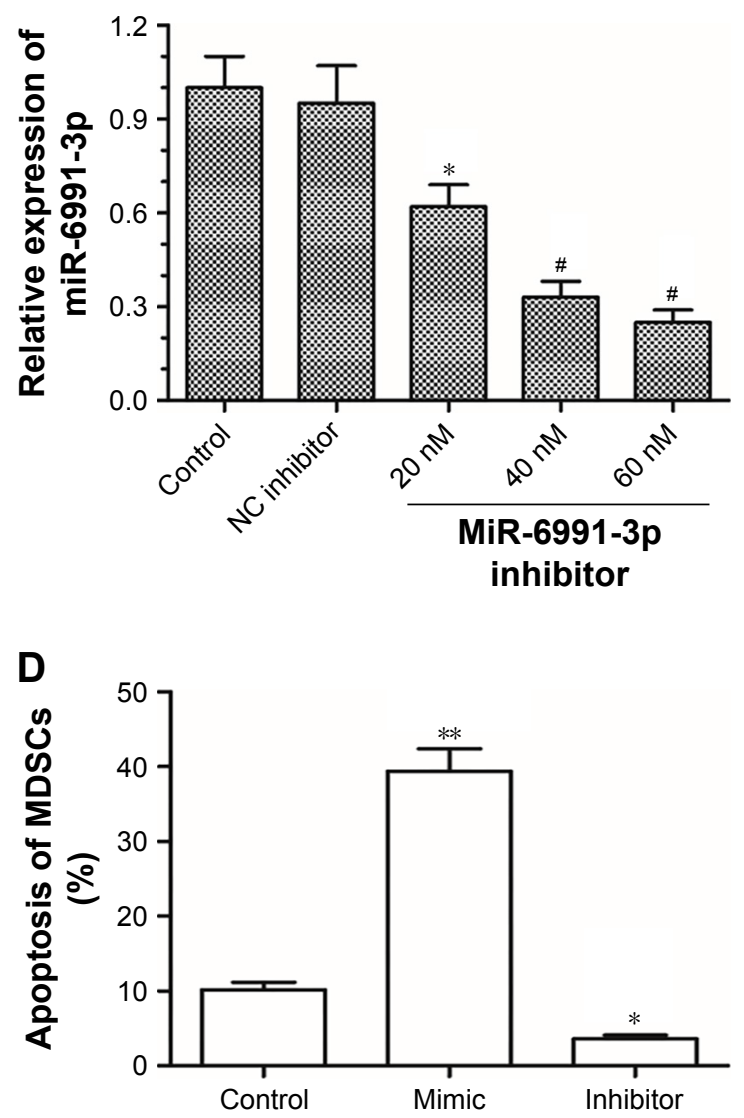

$\mathbf{F}$

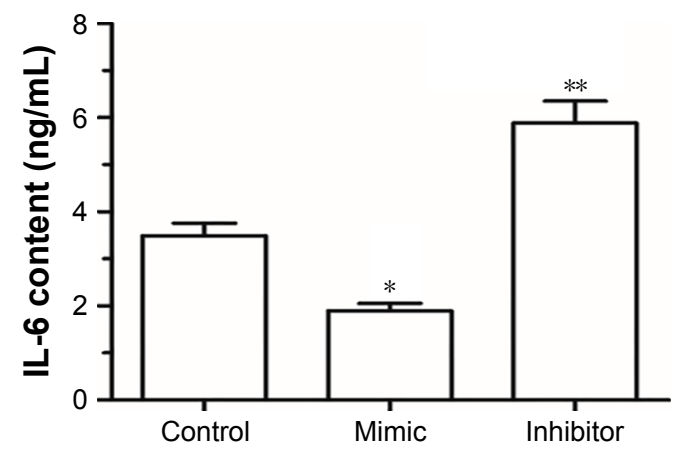

Figure 2 MiR-6991-3p negatively regulated expansion of MDSCs.

Notes: Different concentrations of miR-6991-3p mimic or inhibitor were respectively transfected into the MDSCs. After incubation for 48 hours, the overexpression efficiencies (A) and interference efficiencies (B) were confirmed by qPCR. Amounts of $20 \mathrm{nM}$ miR-699I-3p mimic and $40 \mathrm{nM}$ miR-699I-3p inhibitor were then transfected into MDSCs, and the behaviors of MDSCs were detected, including (C) cell proliferation detected by CCK-8 assay, (D) cell apoptosis determined by PI/Annexin $\vee$ Cell Apoptosis Detection Kit, and secretion of (E) GM-CSF and (F) IL-6 in the supernatant detected with ELISA Kit. $* P<0.05$, $* * P<0.0$ I compared with control, \#P<0.01 compared with control.

Abbreviations: CCK-8, Cell Counting Kit-8; MDSCs, myeloid-derived suppressor cells; NC, negative control.

has been no report revealing the exact role of miR-6991-3p in any biological process. In this study, we found that miR-6991-3p was associated with tumor microenvironment, manifested by decrease in MDSCs from the spleen and further decrease in MDSCs from the tumor tissue. Then, gain- and loss-of-function experiments revealed that miR-6991-3p negatively regulated the expansion, activation, and immunosuppressive effect of MDSCs. Furthermore, we demonstrated that miR-6991-3p could directly target the immune checkpoint gene LGALS9 and suppressed LGALS9-mediated activation 
A

LGALS9 3'UTR 5'-UCUGGCCUAGGGAUAAGGGCUGG-3'

'-GACCUCUUCUUGUUUCC CGACG-5'

B

3'UTR wt 5'-UCUGGCCUAGGGAUAAGGGCUGG-3'

3'UTR mut 5'-UCUGGCCUAGGGAUAAAAACUGG-3'

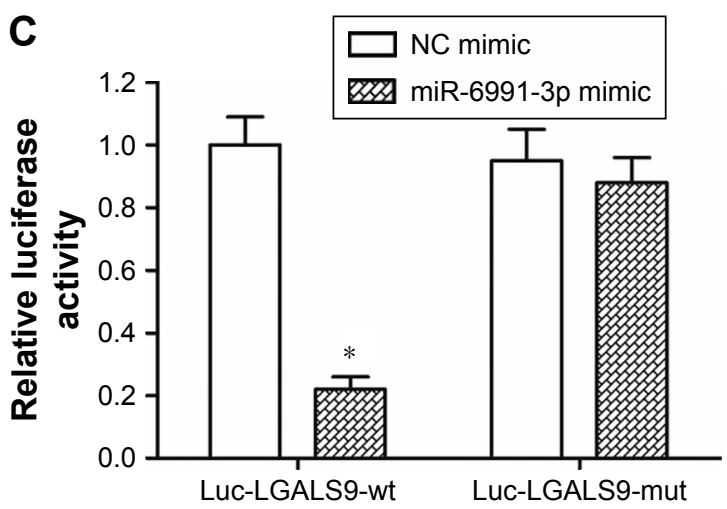

D
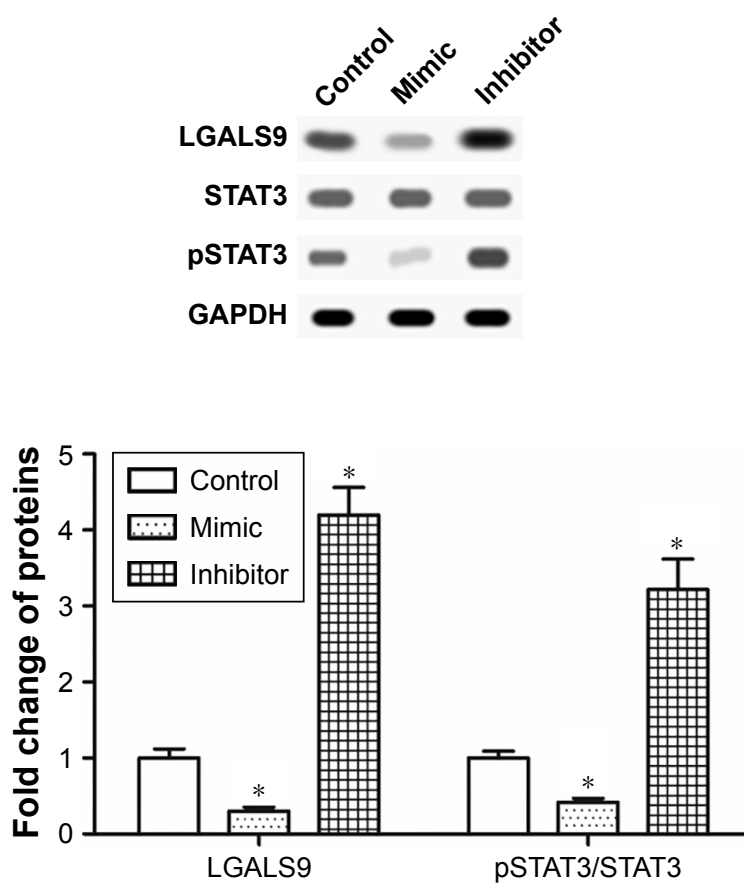

Figure 3 MiR-699I-3p suppressed LAGLS9 translation and STAT3 activation in MDSCs.

Notes: (A) The binding capacity between miR-699I-3p and LAGLS9 mRNA was evaluated by TargetScan. (B) The detailed information about the wt and mut 3'UTR sequence was inserted into the luciferase gene reporter vectors. The red color font represents the mutated bases. (C) Detection of the luciferase activity of wt and mut luciferase gene reporter vectors in response to NC mimic or miR-699I-3p mimic. Amounts of $20 \mathrm{nM}$ miR-699I-3p mimic and $40 \mathrm{nM}$ miR-699I-3p inhibitor were transfected into MDSCs. (D) Expression of LAGLS9, STAT3, and p-STAT3 proteins was detected with Western blotting. $* p<0.05$.

Abbreviations: MDSCs, myeloid-derived suppressor cells; mut, mutated; NC, negative control; wt, wild type.

of STAT3. To our knowledge, this is the first report on the role of miR-6991-3p.

LGALS9 gene encodes the LGALS9 protein which is a $36 \mathrm{kDa}$ beta-galactoside lectin protein. ${ }^{14}$ LGALS9 has been demonstrated to be upregulated in various hematological malignancies and solid tumors, including liver carcinoma. ${ }^{15,16}$ LGALS9 has been identified as an immune checkpoint molecule in many types of cancer. ${ }^{15,17}$ It has been shown that LGALS9 was regulated by Tim-3 and impaired the anticancer activity of natural killer cells. LGALS9 was also demonstrated to suppress the cytotoxic CD8(+) $\mathrm{T}$ cells, memory CD8(+) T cells, and helper T cells. ${ }^{17-19}$ In this study, we reported that LGALS9 was targeted and negatively regulated by miR-6991-3p, resulting in increased activation of STAT3, enhanced expansion and activation of MDSCs, and augmented immunosuppressive effect of MDSCs. Moreover, upregulation of LGALS9 by inhibition of miR-6991-3p displayed the opposite effects. There is several research on the effects of LGALS9 (an LAGLS9 encoding protein) in MDSC $s^{20,21}$ and T cells. ${ }^{22-24}$ LGALS9 induces T cell apoptosis through the CASP1 signaling pathway. Furthermore, LGALS9 may induce the expansion of
CD11b+Ly-6C+MDSCs in hearts of CVB3-infected mice. Hence, it has been confirmed that LGALS9 induces the expansion of MDSCs. Consistent with our results, previous studies also showed that LGALS9 upregulation activated STAT3 and enhanced the immunosuppressive effect of MDSCs. ${ }^{25,26}$

The JAK/STAT pathway is quite an important signaling chain involved in processes such as immunity, cell division, cell death, and tumor formation. The JAK2/STAT3 pathway was recently shown to be involved in expansion and activation of MDSCs. ${ }^{27}$ STAT3-targeted siRNA abrogated the immunosuppressive function of MDSCs and decreased Arg-1 activity. ${ }^{28}$ Blockade of the JAK2/STAT3 pathway has been regarded as an effective measurement for suppression of MDSCs, tumor growth, and tumor-induced angiogenesis. ${ }^{28}$ Studies found that LGALS9 partly mediates the activation of the JNK and p38 MAPK pathways, ${ }^{29}$ and ERK and/or p38 MAPK can mediate IL-6 gene and GM-CSF gene expression. ${ }^{30,31}$ Therefore, miR-6991-3p may regulate the expression of GM-CSF or IL-6 through the LGALS9-p38MAPK axis. Many factors induce expansion and activation of MDSCs, including IL-6, GM-CSF, VEGF, IL-4/10/13, and 
A

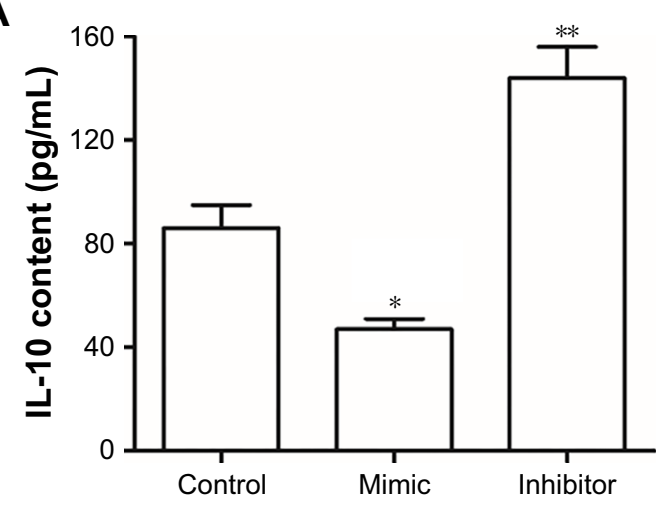

C

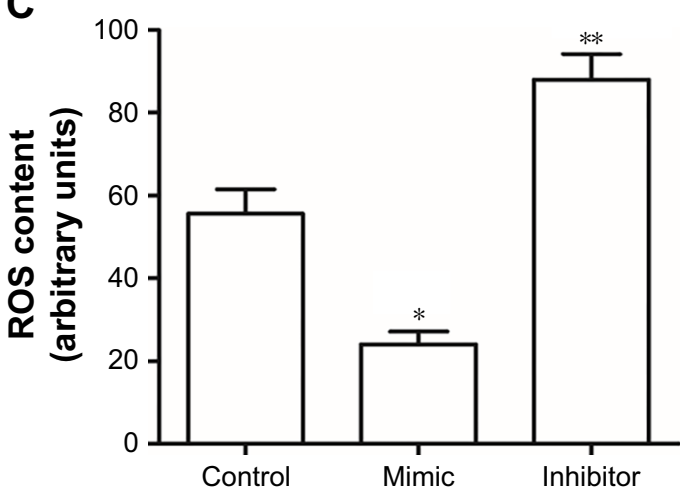

B

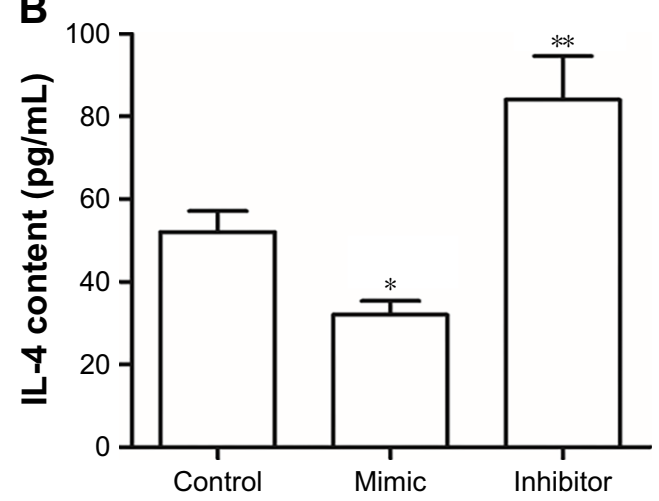

D

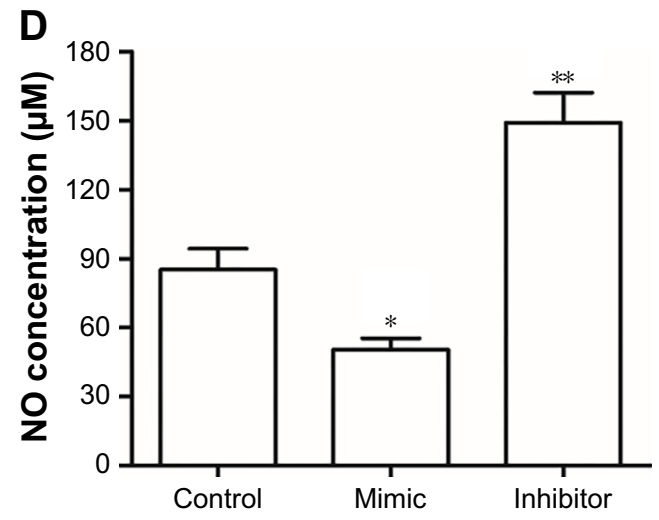

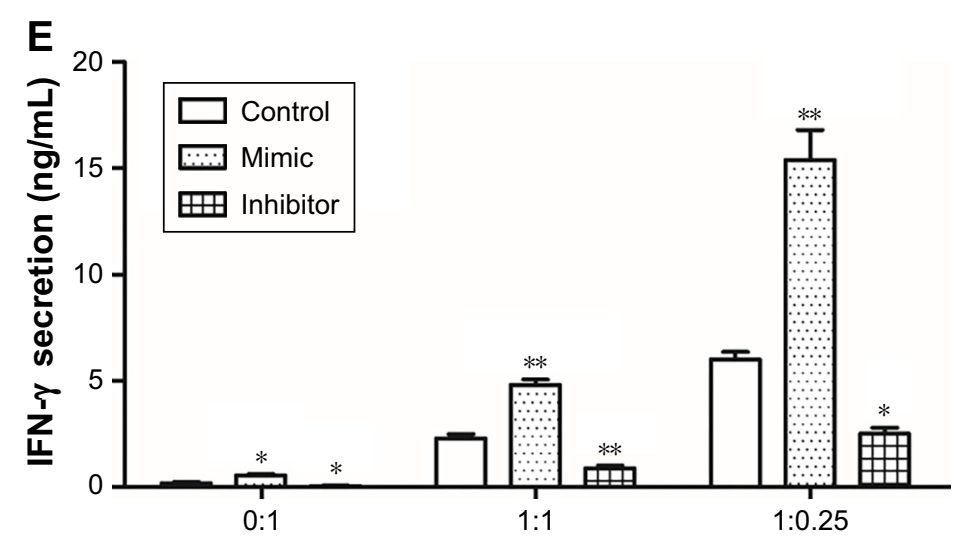

Figure 4 MiR-699I-3p suppressed the activation and immunosuppressive effect of MDSCs.

Notes: Amounts of $20 \mathrm{nM}$ miR-699I-3p mimic and $40 \mathrm{nM}$ miR-699I-3p inhibitor were transfected into MDSCs. After incubation for 48 hours, the secretion of IL-I0 (A) and IL-4 (B) was detected with ELISA Kit, and the production of ROS (C) and NO (D) were detected with corresponding kits. An amount of 200 nM OVA257-264 was co-cultured with MDSCs transfected with various oligos in 96-well plates at a ratio of 0:I, I:I, and I:0.25 for 48 hours. (E) The production of IFN- $\gamma$ was measured by IFN- $\gamma$ ELISA Kit. $* P<0.05, * * P<0.01$ compared with control.

Abbreviation: MDSCs, myeloid-derived suppressor cells.

TGF-beta, etc. ${ }^{32,33}$ On the other hand, our results showed that miR-6991-3p mimic obviously increased cell apoptosis. Therefore, we think that miR-6991-3p influences the expansion and activation of MDSCs by regulating multiple cytokines' expression, not only IL-6. Thus, overexpressing miR-6991-3p and coculturing with hepatocellular carcinoma cells would still block the expansion of MDSCs. In this study, we found that miR-6991-3p negatively regulated LGALS9mediated activation of STAT3 and the immunosuppressive effect of MDSCs.

\section{Conclusion}

miR-6991-3p was downregulated by tumor microenvironment, it targeted the LGALS9/STAT3 pathway, and 
negatively regulated the expansion, activation, and immunosuppressive effect of MDSCs.

\section{Acknowledgment}

This study was supported by the Scientific and Technological Project of Henan Province in China (no 182102310367).

\section{Disclosure}

The authors report no conflicts of interest in this work.

\section{References}

1. Pardoll D. Does the immune system see tumors as foreign or self? Annu Rev Immunol. 2003;21:807-839.

2. Wu L, Yun Z, Tagawa T, et al. Activation of CD1d-restricted natural killer $\mathrm{T}$ cells can inhibit cancer cell proliferation during chemotherapy by promoting the immune responses in murine mesothelioma. Cancer Immunol Immunother. 2014;63(12):1285-1296.

3. Paulos CM, Kaiser A, Wrzesinski C, et al. Toll-like receptors in tumor immunotherapy. Clin Cancer Res. 2007;13(18 Pt 1):5280-5289.

4. Alsina M, Moehler M, Hierro C, Guardeño R, Tabernero J. Immunotherapy for gastric cancer: a focus on immune checkpoints. Target Oncol. 2016;11(4):469-477.

5. Youn JI, Nagaraj S, Collazo M, Gabrilovich DI. Subsets of myeloidderived suppressor cells in tumor-bearing mice. J Immunol. 2008; 181(8):5791-5802.

6. Chandra D, Gravekamp C. Myeloid-derived suppressor cells. Oncoimmunology. 2013;2(11):e26967.

7. Rahat MA, Shakya J, Jivan S. Parallel aspects of the microenvironment in cancer and autoimmune disease. Mediators Inflamm. 2016;2016: $1-17$.

8. Liu D, You M, Xu Y, et al. Inhibition of curcumin on myeloidderived suppressor cells is requisite for controlling lung cancer. Int Immunopharmacol. 2016;39:265-272.

9. Lin Y, Yang X, Liu W, et al. Chemerin has a protective role in hepatocellular carcinoma by inhibiting the expression of IL-6 and GM-CSF and MDSC accumulation. Oncogene. 2017;36(25):3599-3608.

10. Lechner MG, Liebertz DJ, Epstein AL. Characterization of cytokine-induced myeloid-derived suppressor cells from normal human peripheral blood mononuclear cells. J Immunol. 2010;185(4): 2273-2284.

11. Xu Z, Ji J, Xu J, et al. MiR-30a increases MDSC differentiation and immunosuppressive function by targeting SOCS3 in mice with B-cell lymphoma. FEBS J. 2017;284(15):2410-2424.

12. Guo X, Qiu W, Liu Q, et al. Immunosuppressive effects of hypoxiainduced glioma exosomes through myeloid-derived suppressor cells via the $\mathrm{miR}-10 \mathrm{a} /$ Rora and miR-21/Pten pathways. Oncogene. 2018;37(31): 4239-4259.

13. Ladewig E, Okamura K, Flynt AS, Westholm JO, Lai EC. Discovery of hundreds of mirtrons in mouse and human small RNA data. Genome Res. 2012;22(9):1634-1645.

14. Wada J, Kanwar YS. Identification and characterization of galectin-9, a novel beta-galactoside-binding mammalian lectin. J Biol Chem. 1997; 272(9):6078-6086.

15. Gonçalves Silva I, Yasinska IM, Sakhnevych SS, et al. The tim-3galectin-9 secretory pathway is involved in the immune escape of human acute myeloid leukemia cells. EBioMedicine. 2017;22:44-57.

16. Irie A, Yamauchi A, Kontani K, et al. Galectin-9 as a prognostic factor with antimetastatic potential in breast cancer. Clin Cancer Res. 2005; 11(8):2962-2968
17. Fujihara S, Mori H, Kobara H, et al. Galectin-9 in cancer therapy. Recent Pat Endocr Metab Immune Drug Discov. 2013;7(2):130-137.

18. Seki M, Oomizu S, Sakata KM, et al. Galectin-9 suppresses the generation of Th17, promotes the induction of regulatory $\mathrm{T}$ cells, and regulates experimental autoimmune arthritis. Clin Immunol. 2008; 127(1):78-88.

19. Zhu C, Anderson AC, Schubart A, et al. The Tim-3 ligand galectin-9 negatively regulates $\mathrm{T}$ helper type 1 immunity. Nat Immunol. 2005; 6(12):1245-1252.

20. Zhang Y, Zhang M, Li X, Tang Z, He L, Lv K. Expansion of CD11 $b^{+}$Ly- $6 \mathrm{C}^{+}$myeloid-derived suppressor cells (MDSCs) driven by galectin-9 attenuates CVB3-induced myocarditis. Mol Immunol. 2017; 83:62-71.

21. Zhang ZN, Yi N, Zhang TW, et al. Myeloid-Derived Suppressor Cells Associated With Disease Progression in Primary HIV Infection: PD-L1 Blockade Attenuates Inhibition. J Acquir Immune Defic Syndr. 2017; 76(2):200-208.

22. Sehrawat S, Reddy PB, Rajasagi N, Suryawanshi A, Hirashima M, Rouse BT. Galectin-9/TIM-3 interaction regulates virus-specific primary and memory CD8 T cell response. PLoS Pathog. 2010;6(5): e1000882.

23. Seki M, Oomizu S, Sakata KM, et al. Galectin-9 suppresses the generation of Th17, promotes the induction of regulatory $\mathrm{T}$ cells, and regulates experimental autoimmune arthritis. Clin Immunol. 2008;127(1):78-88.

24. Kashio Y, Nakamura K, Abedin MJ, et al. Galectin-9 induces apoptosis through the calcium-calpain-caspase-1 pathway. J Immunol. 2003; 170(7):3631-3636.

25. Ma CJ, Li GY, Cheng YQ, et al. Cis association of galectin-9 with Tim-3 differentially regulates IL-12/IL-23 expressions in monocytes via TLR signaling. PLoS One. 2013;8(8):e72488.

26. Liu JF, Deng WW, Chen L, et al. Inhibition of JAK2/STAT3 reduces tumor-induced angiogenesis and myeloid-derived suppressor cells in head and neck cancer. Mol Carcinog. 2018;57(3):429-439.

27. Thorn M, Guha P, Cunetta M, et al. Tumor-associated GM-CSF overexpression induces immunoinhibitory molecules via STAT3 in myeloid-suppressor cells infiltrating liver metastases. Cancer Gene Ther. 2016;23(6):188-198.

28. Zhang W, Jiang M, Chen J, et al. SOCS3 suppression promoted the recruitment of $\mathrm{CD} 1 \mathrm{~b}^{+} \mathrm{Gr}^{-1} 1^{-} \mathrm{F} 4 / 80^{-} \mathrm{MHCII}^{-}$early-stage myeloid-derived suppressor cells and accelerated interleukin-6related tumor invasion via affecting myeloid differentiation in breast cancer. Front Immunol. 2018;9:1699.

29. Kobayashi T, Kuroda J, Ashihara E, et al. Galectin-9 exhibits antimyeloma activity through JNK and p38 MAP kinase pathways. Leukemia. 2010;24(4):843-850.

30. Sano M, Fukuda K, Sato T, et al. ERK and p38 MAPK, but not NFkappaB, are critically involved in reactive oxygen species-mediated induction of IL-6 by angiotensin II in cardiac fibroblasts. Circ Res. 2001; 89(8):661-669.

31. Vordenbäumen S, Braukmann A, Petermann K, et al. Casein $\alpha \mathrm{s} 1$ is expressed by human monocytes and upregulates the production of GM-CSF via p38 MAPK. J Immunol. 2011;186(1):592-601.

32. Lin Y, Yang X, Liu W, et al. Chemerin has a protective role in hepatocellular carcinoma by inhibiting the expression of IL-6 and GM-CSF and MDSC accumulation. Oncogene. 2017;36(25):3599-3608.

33. Lechner MG, Liebertz DJ, Epstein AL. Characterization of cytokineinduced myeloid-derived suppressor cells from normal human peripheral blood mononuclear cells. J Immunol. 2010;185(4):2273-2284. 


\section{Publish your work in this journal}

OncoTargets and Therapy is an international, peer-reviewed, open access journal focusing on the pathological basis of all cancers, potential targets for therapy and treatment protocols employed to improve the management of cancer patients. The journal also focuses on the impact of management programs and new therapeutic agents and protocols on
Dovepress

patient perspectives such as quality of life, adherence and satisfaction. The manuscript management system is completely online and includes a very quick and fair peer-review system, which is all easy to use. Visit http://www.dovepress.com/testimonials.php to read real quotes from published authors.

Submit your manuscript here: http://www.dovepress.com/oncotargets-and-therapy-journal 